\title{
A technique for minimally altering anatomically based subthalamic electrode targeting by microelectrode recording
}

\author{
Patrick B. Senatus, M.D., Ph.D., David Teeple, M.D., Shearwood McClelland III, M.D., \\ Seth L. Pullman, M.D., F.R.C.P.(C), QiPing Yu, Ph.D., Blair Ford, M.D., F.R.C.P.(C), \\ Guy M. McKhann II, M.D., ANd Robert R. Goodman, M.D., Ph.D.
}

\author{
Departments of Neurological Surgery and Neurology, Neurological Institute of New York, Columbia \\ University College of Physicians and Surgeons, New York, New York; Department of Neurology, \\ Barrow Neurological Institute, Phoenix, Arizona; and Department of Neurosurgery, University of \\ Minnesota Medical School, Minneapolis, Minnesota
}

\begin{abstract}
Object. Implantation of a subthalamic nucleus (STN) deep brain stimulation (DBS) electrode is increasingly recognized as an effective treatment for advanced Parkinson disease (PD). Despite widespread use of microelectrode recording (MER) to delineate the boundaries of the STN prior to stimulator implantation, it remains unclear to what extent MER improves the clinical efficacy of this procedure. In this report, the authors analyze a series of patients who were treated at one surgical center to determine to what degree final electrode placement was altered, based on readings obtained with MER, from the calculated anatomical target.

Methods. Subthalamic DBS devices were placed bilaterally in nine patients with advanced PD. Frame-based volumetric magnetic resonance images were acquired and then transferred to a stereotactic workstation to determine the anterior and posterior commissure coordinates and plane. The initial anatomical target was $4 \mathrm{~mm}$ anterior, $4 \mathrm{~mm}$ deep, and $12 \mathrm{~mm}$ lateral to the midcommissural point. The MERs defined the STN boundaries along one or more parallel tracks, refining the final electrode placement by comparison of results with illustrations in a stereotactic atlas.

In eight of 18 electrodes, the MER results did not prompt an alteration in the anatomically derived target. In another eight placements, MER altered the target by less than $1 \mathrm{~mm}$ and two of 18 electrode positions differed by less than $2 \mathrm{~mm}$. The anterior-posterior difference was $0.53 \pm 0.65 \mathrm{~mm}$, whereas the medial-lateral direction differed by $0.25 \pm 0.43 \mathrm{~mm}$. The ventral boundary of the STN defined by MER was $2 \pm 0.72 \mathrm{~mm}$ below the calculated target (all values are the means \pm standard deviation). All patients attained clinical improvement, similar to previous reports.

Conclusions. In this series of patients, microelectrode mapping of the STN altered the anatomically based target only slightly. Because it is not clear whether such minor adjustments improve clinical efficacy, a prospective clinical comparison of MER-refined and anatomical targeting may be warranted.
\end{abstract}

\section{KEY WORDS • deep brain stimulation • subthalamic nucleus • microelectrode recording • Parkinson disease}

\section{Use of DBS for PD}

Patients with advanced idiopathic PD exhibit the disabling motor signs of tremor, rigidity, and bradykinesia as well as axial symptomatology including freezing, gait difficulty, and postural instability. ${ }^{5,15}$ In patients in whom PD is medically refractory, stereotactic localization and inactivation of the STN by using continual high-frequency DBS is increasingly becoming the treatment of choice because of its demonstrated safety and efficacy. ${ }^{6,13,14}$ Compared with nuclear ablation, DBS offers the advantages of reversibility and modulation, and can be tailored to a patient's clinical status. ${ }^{1,3,4,12}$

Abbreviations used in this paper: $\mathrm{AC}-\mathrm{PC}=$ anterior commissure-posterior commissure; AP = anterior-posterior; DBS = deep brain stimulation; $\mathrm{MER}=$ microelectrode recording; $\mathrm{ML}=$ medial-lateral; $\mathrm{MR}=$ magnetic resonance; $\mathrm{PD}=$ Parkinson disease; STN = subthalamic nucleus.

\section{Localization of the STN}

The efficacy of STN inactivation is presumably related to the precision with which the DBS electrode is placed within the nucleus. Stimulating electrode tips are $1.27 \mathrm{~mm}$ in diameter and the STN is ovoid, of modest dimensions $(7 \times 9 \times 5 \mathrm{~mm})$, and obliquely oriented. Precise targeting to the center of the STN is thought to optimize clinical outcome. To this end, direct and indirect anatomical techniques have been used, usually coupled with corroborative physiological MER. ${ }^{2,18}$

Indirect targeting relies on a human anatomical atlas based on the horizontal plane that runs through the AC and PC. The position of the deep nuclei relative to the AC-PC line is obtained from the atlas. Computerized tomography or MR images obtained with the aid of a frame secured to the patient's head are used to ascertain the $\mathrm{x}, \mathrm{y}$, and $\mathrm{z}$ coordinates of the commissure and then to calculate the coordinates of the STN relative to the ACPC line. Direct targeting requires visualization of the STN 
on coronal or axial $\mathrm{T}_{2}$-weighted or inversion-recovery MR sequences, usually assisted by calculation of the STN coordinates from the AC-PC line, as with the indirect method.

Extensive experience at our institution with stereotactic lesioning and DBS electrode implantation for movement disorders (primarily targeting the ventral intermediate nucleus of the thalamus and the medial segment of the globus pallidus) supports the suggestion that calculating the target relative to the AC-PC line alone usually obtains a mark that is within $2 \mathrm{~mm}$ of the desired physiological target. The goal of DBS electrode implantation is placement of the device through the center of the motor portion of the STN. In many medical centers, MER is used to identify the boundaries of the STN physiologically, with the goal of improving on anatomical imaging alone. It is not yet clear to what degree the desired clinical outcome is determined by the proximity of the electrode to the center of the STN, or in what percentage of implants the MER findings significantly alter the target.

\section{Study Design}

In this report, we retrospectively analyze to what extent the final electrode target was altered from the calculated indirect anatomical target by MER findings in nine patients who underwent bilateral STN implantation of DBS electrodes.

\section{Clinical Material and Methods}

\section{Patient Demographic Data}

The surgical records of nine consecutive patients who underwent bilateral STN placement of a DBS electrode (model 3387 or 3389; Medtronic, Inc., Minneapolis, MN) at our institution in a 10-month period (July 1999-May 2000) were retrospectively examined to determine the difference between the anatomically calculated and final DBS electrode target. Patients underwent extensive preoperative evaluation, including detailed neurological examination and volumetric MR imaging of the brain.

\section{Stereotactically Guided Anatomical Targeting}

A functional Cosman-Roberts-Wells head frame (Radionics, Inc., Burlington, MA) was used for stereotactic surgery. During frame placement, roll and yaw were minimized by the use of ear bars. The base ring was aligned to the AC-PC line by parallel angulation to an imaginary line connecting the external auditory meatus and the orbital floor.

Using the StereoPlan (Radionics, Inc.) platform for stereotactic planning, an anatomical target $4 \mathrm{~mm}$ posterior, 4 $\mathrm{mm}$ deep, and $12 \mathrm{~mm}$ lateral to the midcommissural point was selected using manual calculation. Corrections were made for brain tilt and/or rotation relative to the AC-PC plane.

\section{Neurophysiological Localization}

Tungsten impedance monitoring probes that were 24 $\mathrm{mm}$ long with a tip size of 20 to $25 \mu \mathrm{m}$ were advanced to $30 \mathrm{~mm}$ above the calculated anatomical target. Impedance at $1000 \mathrm{~Hz}$ was measured at 3 and $20 \mathrm{~mm}$ beyond the microelectrode's point of exit from the cannula. Subsequently, the recording microelectrode was advanced to the target by using an electronic stepper microdrive, and the electrical signals from single units and background activity were filtered at 100 to $3000 \mathrm{~Hz}$, preamplified and amplified to a total of 10,000 times, digitized, and sent to an oscilloscope and audio system for real-time monitoring, and to a recording device for offline analysis. The MERbased coordinate positions, discharge frequencies, and action potential morphological features of single units, plus fiber activity and background changes were quantified and recorded.

Typically, bursting cells of the anterior thalamus are encountered, followed by a quiet region corresponding to the fields of Forel and zona incerta. Proceeding ventrally, an area of increased background noise and irregularly firing neurons that are often responsive to movement are detected; these correspond to the STN. Farther ventrally below the STN, a region of more rapidly and regularly firing neurons corresponding to the substantia nigra pars reticulata is detected. The mean thickness of the STN (at its greatest extent as determined for each implanted side) found with this method was $5.11 \pm 0.63 \mathrm{~mm}$ (range 3.4$5.8 \mathrm{~mm}$ ). The vertical diameter of the STN and the superior border of the substantia nigra pars reticulata were compared with those expected based on the stereotactic atlas. ${ }^{17}$ If these data indicated that the trajectory was within a substantial portion of the STN (in the ML and AP planes), then this trajectory would be used for DBS electrode placement, with the deepest contact targeted for the ventral STN border.

In every patient, one or two more parallel MER tracks were obtained. The MER results were used to determine the targeting for the DBS electrode (this is discussed further in the Results section). The number of MER tracks used for the first side ranged from two to five, with the majority of patients having two tracks on this side (only two of nine patients had more than three MER tracks). When second and third microelectrode tracks were placed, they were anterior and/or lateral to the initial one (maximum AP deviation, $5 \mathrm{~mm}$ anterior; maximum ML deviation, $4.5 \mathrm{~mm}$ lateral). This allowed us to confirm localization well within the STN in all 18 sides. We had more confidence in ML (range $1 \mathrm{~mm}$ medial to $1 \mathrm{~mm}$ lateral) than in AP targeting (range $1.5 \mathrm{~mm}$ posterior to $2 \mathrm{~mm}$ anterior) because of our concern about AP brain shift due to the head positioning required for surgery.

In every patient with multiple tracks, the first track (defined by the calculated target) was the longest one within the STN, enabling us to use subsequent tracks to confirm targeting at a significant distance from the anterior and lateral STN. Four of the patients underwent staged procedures, whereas in the other five both sides were implanted on the same day. For the patients who underwent staged procedures, the implant was done in the same manner on the second side as on the first (always with at least two MER tracks). For the patients who underwent simultaneous implantation, the procedures were performed using a mirror image (except in one patient whose second side was $1 \mathrm{~mm}$ medial to the first side). Test stimulation (up to $4 \mathrm{~V}$ ) with the DBS electrode yielded no significant sustained side effects and usually produced some improvement in contralateral rigidity and bradykinesia. 


\section{Effects of MER on DBS electrode targeting in the STN}

\section{Postoperative Assessment}

Postoperative evaluation consisted of neurological examination, follow-up MR imaging, and review of PD symptoms and medications. Clinical outcome at more than 6 months postsurgery was measured by responses to a mailed or telephone-administered questionnaire. ${ }^{16} \mathrm{~Pa}$ tients were asked to assess and compare the extent of their preoperative and postoperative symptoms, their skill levels in a number of common activities, and overall functional status, in "on" and in "off" stimulation periods. The symptoms assessed included falling, freezing, tremor, the presence of significant dyskinesias, and sensory complaints. The activities assessed included speech, salivation, swallowing, handwriting, cutting food, dressing, hygiene, turning in bed, walking, and overall activities of daily living. The duration of on and severe off periods was investigated.

\section{Results}

\section{Target Adjustments Based on MER Findings}

Nine patients underwent bilateral placement of DBS electrodes in the STN. For the first MER track in each of the staged bilateral implants and for the first side of the simultaneously placed bilateral implants, the length of the electrode in the STN was at least $4.2 \mathrm{~mm}$ (range 4.2-5.8 $\mathrm{mm}$ ). For the five simultaneous bilateral implants, the first MER track was made on the second side at the mirror image of the DBS target on the first side (range 4.3-5.5 $\mathrm{mm}$ ). In these five implants, only a single MER track was made. In the implants with more than one MER track, the first track identified the longest length of STN in all cases. In eight (44.4\%) of 18 electrodes, the anatomically and/or image-determined target coordinates, in either the AP (y) or ML (x) dimensions were not altered based on MER results. In eight $(44.4 \%)$ of the 18 electrodes, the targets were determined using the two methods differed by $1 \mathrm{~mm}$ or less in either direction. The remaining two placements differed by $2 \mathrm{~mm}$ or less in either direction. The difference was $0.53 \pm 0.65 \mathrm{~mm}$ in the AP (y) dimension and $0.25 \pm$ 0.43 in the ML (x) dimension (all values are the means \pm standard deviation). This translates to a calculated mean discrepancy of $0.59 \mathrm{~mm}$ in the xy plane. The ventral boundary (z) of the STN defined by MER was $2 \pm 0.72 \mathrm{~mm}$ below the calculated target. The final electrode placement relative to the calculated target ranged from -1.5 to $2 \mathrm{~mm}$ in the AP dimension, and from -1 to $1 \mathrm{~mm}$ in the ML dimension, with 16 of 18 electrodes placed precisely at the anatomically and/or image-determined ML target coordinate.

Final microelectrode placement in a patient's contralateral side differed from its mirror image by $0.61 \pm 0.86$ $\mathrm{mm}$ in the AP direction and by $0.28 \pm 0.44 \mathrm{~mm}$ in the ML direction. In six of nine patients, the two sides coincided in their final AP (y) coordinate, and in five of nine patients the sides coincided in their final ML (x) coordinate. Of the nine patients, the final electrode placements were exact mirror images in the xy plane in three cases. The mean difference in STN depth (z) between the right and left sides was $0.85 \pm 0.77$, ranging from 0.2 to $2.2 \mathrm{~mm}$. In five of nine patients, this difference was $0.4 \mathrm{~mm}$ or less. Of the five patients who underwent bilateral implantation on the same day, in four the second electrode was implanted at a mirror image to the first one, and the second electrode in the fifth patient was placed $1 \mathrm{~mm}$ medial to the first electrode.

\section{Clinical Outcome}

Postoperatively, patients demonstrated significant reductions in off motor scores, duration of off episodes, dyskinesia severity, and medication requirements (levodopa equivalents). Simultaneously, the patients had a significant increase in the duration of on time. These changes were similar to those seen in other patients who underwent electrode implantation with this surgical approach, and has been reported in greater detail in previous articles. ${ }^{7,8}$ The clinical improvements we found are similar to those reported by others. ${ }^{11,13,14}$

\section{Discussion}

The objective of this study was to examine the extent to which MER was used to alter DBS electrode placement from the anatomically and/or image-calculated target. Anatomical targeting supplemented by MER is the widely used method for DBS placement in the STN. ${ }^{6,13,14}$ The use of MER is widely presumed to improve the localization of the optimal STN target; however, the degree to which this improves clinical outcome has not yet been rigorously examined..$^{3,18}$ The use of MER increases operating time and the number of electrode penetrations of the brain (presumably increasing the risk of morbidity), and requires expensive additional equipment and neurophysiological expertise. ${ }^{9,10}$

The mean target adjustments made in the AP (y; 0.61 $\mathrm{mm}$ ) and ML (x;0.28 mm) dimensions in this group of patients were small and did not exceed $2 \mathrm{~mm}$ for any electrode. In all implants, the first MER track identified at least $4.2 \mathrm{~mm}$ of STN (range $4.2-5.8 \mathrm{~mm}$ ) so that the subsequent MER tracks, when they were done, served to confirm that the initial track was a significant distance from the anterior and lateral borders of the STN. The adjustments in the $\mathrm{z}$ plane were somewhat greater (mean $2 \mathrm{~mm}$ ), but are of less clinical relevance, because the presence of four contacts along the length of the electrode decreases the importance of the $\mathrm{z}$ plane target accuracy. A similar degree of target adjustment $(1.5 \pm 0.8 \mathrm{~mm})$ based on MER findings has been reported previously. ${ }^{19}$ The clinical significance of this target adjustment has yet to be rigorously determined.

The small number of patients in this series had significant clinical improvements similar to those in other patients who have undergone electrode implantation via the same surgical approach ${ }^{7,16}$ and other approaches that use MER..$^{11,13,14}$ It has not yet been clearly demonstrated that any of the target adjustments made in these patients has resulted in a greater clinical benefit than would be achieved by electrode implantation in the initially calculated target. In fact, there is some evidence that actual DBS electrode location usually varies to some extent (mean $1.3 \mathrm{~mm}$ in the ML and 0.65 in the AP dimension) from the intended coordinates. ${ }^{16}$ This unintended variation has not been clearly related to clinical efficacy. The limited experience reported here does not conclusively demon- 
strate a lack of clinical benefit in the use of MER to modify anatomically and/or image-based targeting. Nevertheless, the target adjustments that were made in this study were of relatively small magnitudes. We have to consider the possibility that these small-magnitude changes may not alter clinical benefit. Other studies in which investigators have looked at the unintended variation of the electrode position from its target have not shown decreased clinical benefit at variations of up to $3 \mathrm{~mm}$ from the intended target. It is possible that even the small adjustments made based on MER results help to avoid the decrease in clinical benefit that might occur because of unintended inaccuracy, but this concern can only be adequately addressed by a prospective study in which image-guided DBS implantation is compared with MER-adjusted procedures.

\section{Acknowledgement}

We would like to thank Irfan Qureshi, M.D., for his contribution to this article..

\section{Disclosure}

None of the authors received any financial support in conjunction with the generation of this submission.

\section{References}

1. Benazzouz A, Boraud T, Féger J, et al: Alleviation of experimental hemiparkinsonism by high-frequency stimulation of the subthalamic nucleus in primates: a comparison with L-Dopa treatment. Mov Disord 11:627-632, 1996

2. Benazzouz A, Breit S, Koudsie A, et al: Intraoperative microrecordings of the subthalamic nucleus in Parkinson's disease. Mov Disord 17 (Suppl 3):S145-S149, 2002

3. Benazzouz A, Gross C, Féger J, et al: Reversal of rigidity and improvement in motor performance by subthalamic high-frequency stimulation in MPTP-treated monkeys. Eur $\mathbf{J}$ Neurosci 5:382-389, 1993

4. Bergman H, Wichmann T, DeLong MR: Reversal of experimental parkinsonism by lesions of the subthalamic nucleus. Science 249:1436-1438, 1990

5. Cotzias GC, Van Woert MH, Schiffer LM: Aromatic amino acids and modification of parkinsonism. N Eng J Med 276: 374-379, 1967

6. The Deep-Brain Stimulation for Parkinson's Disease Study Group: Deep-brain stimulation of the subthalamic nucleus or the pars interna of the globus pallidus in Parkinson's disease. $\mathbf{N}$ Engl J Med 345:956-963, 2001
7. Ford B, Winfield L, Pullman SL, et al: Subthalamic nucleus stimulation in advanced Parkinson's disease: blinded assessments at one year follow up. J Neurol Neurosurg Psychiatry 75:1255-1259, 2004

8. Goodman RR, Kim B, McClelland S III, et al: Operative techniques and morbidity with subthalamic nucleus deep brain stimulation in 100 consecutive patients with advanced Parkinson's disease. J Neurol Neurosurg Psychiatry 77:12-17, 2006

9. Hariz MI, Fodstad H: Do microelectrode techniques increase accuracy or decrease risks in pallidotomy and deep brain stimulation? A critical review of the literature. Stereotact Funct Neurosurg 72:157-169, 1999

10. Honey CR, Berk C, Palur RS, et al: Microeclectrode recording for pallidotomy: mandatory, beneficial or dangerous? Stereotact Funct Neurosurg 77:98-100, 2001

11. Just H, Ostergaard K: Health-related quality of life in patients with advanced Parkinson's disease treated with deep brain stimulation of the subthalamic nuclei. Mov Disord 17:539-545, 2002

12. Krack P, Pollak P, Limousin P, et al: Subthalamic nucleus or internal pallidal stimulation in young onset Parkinson's disease. Brain 121:451-457, 1998

13. Kumar R, Lozano AM, Kim YJ, et al: Double-blind evaluation of subthalamic nucleus deep brain stimulation in advanced Parkinson's disease. Neurology 51:850-855, 1998

14. Limousin P, Krack P, Pollak P, et al: Electrical stimulation of the subthalamic nucleus in advanced Parkinson's disease. N Engl J Med 339:1105-1111, 1998

15. Marsden CD, Parkes JD: "On-off" effects in patients with Parkinson's disease on chronic levodopa therapy. Lancet 1: 292-296, 1976

16. McClelland S III, Ford B, Senatus PB, et al: Subthalamic stimulation for Parkinson disease: determination of electrode location necessary for clinical efficacy. Neurosurg Focus 19(5): E12, 2005

17. Schaltenbrand G, Bailey P: Introduction to Stereotaxis, with an Atlas of the Human Brain. Stuttgart: Thieme, 1959

18. Starr PA, Vitek JL, Bakay RAE: Deep brain stimulation for movement disorders. Neurosurg Clin N Am 9:381-402, 1998

19. Zonenshayn M, Rezai AR, Mogilner AY, et al: Comparison of anatomic and neurophysiological methods for subthalamic nucleus targeting. Neurosurgery 47:282-294, 2000

Manuscript received January 6, 2006.

Accepted in final form March 31, 2006.

Address reprint requests to: Robert R. Goodman, M.D., Ph.D., Neurological Institute of New York, Department of Neurological Surgery, 710 West 168th Street, Box 99, New York, New York 10032. email: rrg2@columbia.edu. 